\title{
Efetividade de selantes de fossas e fissuras aplicados por estudantes de Odontologia na prevenção de cárie
}

\section{Effectiveness of pit and fissure sealants applied by Dental students for dental caries prevention}

\author{
Viviane Silveira Marques* \\ Guilhermo Enrico Sartori de Oliveira Andres* \\ Daniela de Rossi Figueiredo ${ }^{* * *}$
}

\section{Resumo}

Objetivo: avaliar a efetividade dos selantes aplicados por estudantes do curso de graduação em Odontologia da Universidade do Sul de Santa Catarina (Unisul), Grande Florianópolis, após 6 meses da aplicação, e estimar a prevalência de cárie dos participantes. Sujeitos e método: estudo observacional descritivo com indivíduos das clínicas de Odontologia. A efetividade dos selantes oclusais foi avaliada por roteiro validado na literatura, e a prevalência de cárie, dicotomizada. Os dados foram analisados por estatística descritiva e as associações pelo teste Qui-Quadrado. Resultados: a taxa de resposta do estudo foi de $94,7 \% \quad(n=18), 2 / 3$ da amostra eram do sexo feminino (72,2\% IC 95\% $49,1 ; 87,5), 60 \%$ dos pacientes recebiam 3 salários mínimos ou menos, maiores proporções de indivíduo tinham selados seus dentes há pelo menos 9 meses. Pouco menos de $2 / 3$ da amostra receberam Fluroshield como material restaurador em seus dentes. Dentre os 57 dentes avaliados, 70,2\% apresentavam retenção total. Os dentes mais acometidos pela perda de retenção foram os segundos molares superiores (40\%). Mais de $2 / 3$ da amostra não apresentaram cárie (88,9\% IC 95\% $67,2 ; 96,9)$. O isolamento relativo foi o mais prevalente $(72,2 \%$ IC 95\% 49,1;87,5). Conclusão: sob a perspectiva das práticas dos estudantes de Odontologia, metade da amostra de pacientes apresentou alguma perda de retenção dos selantes, mesmo que um número maior de retenções totais e a ausência de recidiva de cárie tenham sido observados. Isso reflete a importância da relação professor-aluno no planejamento e na correta aplicação das técnicas de selamento por estudantes de Odontologia.

Palavras-chave: Efetividade. Saúde bucal. Selantes de fossas e fissuras. Suscetibilidade à cárie dentária.

\section{Introdução}

Apesar do declínio de cárie nas populações nas últimas décadas, o componente cariado ainda é o mais prevalente entre crianças e adolescentes brasileiros. Aos 5 anos de idade, uma criança brasileira tem, em média, 2,43 dentes com experiência de cárie, sendo o componente cariado responsável por $80 \%$ do índice de dentes cariados, perdidos e obturados (CPO-D). Ainda segundo o inquérito nacional, para a experiência de cárie, mais da metade das crianças brasileiras, aos 5 e 12 anos, em 2010, já tinham tido alguma experiência de cárie, e este número aumenta para $76 \%$ entre os adolescentes ${ }^{1}$.

Todos os indivíduos portadores de dentes naturais são susceptíveis à cárie dentária, e maiores suscetibilidades à cárie são observadas nos primeiros molares $^{2-4}$. Da mesma maneira, a experiência de cárie durante a dentição decídua é considerada como um forte preditor da doença na dentição permanen$t^{5}$. Um estudo longitudinal realizado por Skeie et al. ${ }^{6}$ (2006) demonstrou que superfície de molares decíduos com experiência de cárie, em crianças aos 5 anos de idade, foi importante preditora clínica de alto risco de cárie aos 10 anos das mesmas crianças.

Não obstante, a literatura demonstra a importância dos cuidados preventivos em odontologia, em especial dos molares permanentes, pela anatomia de fossas e fissuras e maior tempo de exposição em boca e, dentre os métodos preventivos disponíveis, destacam-se os selantes com o status de principal método preventivo para aplicação profissional em fóssulas e fissuras dentais ${ }^{7,8}$. Revisões sistemáticas têm assegurado a eficácia dos selantes dentários de

Cirurgiã-dentista, Palhoça, Santa Catarina, Brasil

Acadêmico do Curso de Odontologia, Universidade do Sul de Santa Catarina, Palhoça, Santa Catarina, Brasil.

Professora da Graduação em Odontologia, Universidade do Sul de Santa Catarina, Palhoça, Santa Catarina, Brasil. 
fossas e fissuras na prevenção da cárie oclusal de dentes permanentes ${ }^{3,9}$. Estudos que avaliaram o nível de contaminação bacteriana antes e após o uso de selantes demonstraram redução expressiva do número de bactérias metabolicamente viáveis após o uso de selamento ${ }^{10}$. Selantes ionoméricos foram efetivos na retenção e prevenção de lesões cariosas no período de 6 meses ${ }^{11}$.

A retenção é um dos pré-requisitos mais importantes de selantes de fossas e fissuras, quanto mais tempo o material permanece ligado à superfície do dente, mais eficaz será a sua ação protetora ${ }^{12}$. Da mesma maneira, a avaliação da destreza e da efetividade na aplicação da técnica é um requisito importante, embora apenas um estudo sobre a avaliação dos selantes por estudantes foi encontrado ${ }^{13}$.

Considerando a prevalência de cárie em dentes permanentes de crianças e adolescentes brasileiros, a retenção dos selantes como método preventivo e a importância da efetividade na aplicação dos selantes, este estudo teve como objetivo avaliar a efetividade dos selantes, quando da aplicação por estudantes de Odontologia, após, pelo menos, 6 meses da aplicação, assim como estimar a prevalência de cárie dos participantes.

\section{Sujeitos e método}

Estudo observacional, descritivo e de caráter transversal com amostra do tipo não probabilística e intencional com indivíduos que receberam selante em dentes permanentes, durante o atendimento pelos estudantes nas clínicas do curso de graduação em Odontologia da Universidade do Sul de Santa Catarina (Unisul), localizada no município de Palhoça, Santa Catarina. O presente trabalho foi submetido ao Comitê de Ética em Pesquisa da Unisul (CEP-Unisul) e aprovado sob o parecer 1.824.523.

Foram incluídos todos os indivíduos, independente de faixas etárias, que receberam o selante em dentes permanentes há 6 meses, excluindo-se os prontuários que não continham informações adequadas sobre tempo de aplicação, tipo de material ou isolamento realizado.

Dos 1.419 prontuários disponíveis, 19 indivíduos com registro adequado e que receberam selante há 6 meses foram identificados. Um contato telefônico e um convite para retorno à clínica, em horários diversificados estabelecidos pela pesquisadora, foram feitos aos indivíduos, assim como a garantia de avaliação e o encaminhamento para as necessidades de tratamento.

Técnicas de aplicação dos selantes e materiais disponíveis no curso de Odontologia da Unisul Grande Florianópolis

A técnica de aplicação do selante inicia-se pela limpeza dos dentes. Após o diagnóstico da gravidade da cárie, utiliza-se a técnica não invasiva ou minimamente invasiva. Isolamento absoluto ou relativo pode ser utilizado e, em geral, é selecionado de acordo com as condições de trabalho e a opção do material restaurador ${ }^{8,14}$. A inserção do material segue a preconização dos fabricantes. Os materiais disponíveis na clínica escola da Unisul são: Fluroshield, Resina Fluída Natural Flow - DFL, Prevent - FGM e Vitremer. O fotoativador disponível é o FOTO OPTILIGHT LD MAX 100/240V-50/60HZ, da marca GNATUS; possui luz de LED com comprimento de onda da luz na faixa entre $440 \mathrm{~nm}$ e $460 \mathrm{~nm}(1.200 \mathrm{~mW} / \mathrm{cm} 2$ de potência de luz).

\section{Instrumentos de avaliação utilizados e logística da avaliação}

A efetividade dos selantes oclusais seguiu o roteiro de Basting et al. ${ }^{15}$ (1999), que preconiza avaliar retenção total (RT): total permanência do selante sobre a superfície oclusal, sem haver mínima perda do material; retenção parcial tipo $1(\mathrm{R} 1)$ : presença do selante em $2 / 3$ da extensão dos sulcos, observando-se pequenas fraturas e perdas do material; retenção parcial tipo 2 (R2): presença do selante em 1/3 da extensão dos sulcos, observando-se maiores fraturas e perdas do material; perda total (PT): ausência do material selador sobre a superfície oclusal dos dentes.

Há 6 meses da aplicação, os dentes foram limpos com escova Robson e água antes do início das avaliações. A sonda ball point, recomendada pela Organização Mundial da Saúde, foi utilizada para avaliação, assim como para retenção de acúmulo adicional de placa. Antes do exame, procedeu-se à secagem do dente com rolete de algodão e/ou ar comprimido, para os casos de dúvida. Apenas a pesquisadora fez a análise dos padrões de retenção. A iluminação foi feita pelo refletor em consultório odontológico da Unisul. Foi avaliada, também, a presença de cárie pelo índice ICDAS $\mathrm{II}^{16,17}$ e estimada a prevalência de cárie dicotomizada em presença ou ausência nos dentes selados.

\section{Análise estatística}

Os dados coletados são apresentados em tabelas de contingência segundo estatística descritiva. O desfecho utilizado foi alguma perda de retenção ( $\operatorname{sim} /$ não), que incluiu as perdas parciais e totais dos dentes selados. Foram testadas associações entre o desfecho e as variáveis sociodemográficas sexo, idade e renda, além do tipo de isolamento, material restaurador e prevalência de cárie. Paras as associações, foi utilizado o teste Qui-Quadrado. A significância estatística utilizada foi $\mathrm{p} \leq 0,05$. 


\section{Resultados}

A taxa de resposta do estudo foi de $94,7 \%$ ( $n=18$ ). Segundo dados descritivos, $2 / 3$ da amostra eram do sexo feminino (72,2\% IC 95\% 49,1;87,5). Em relação à renda, $60 \%$ dos pacientes recebiam 3 salários mínimos ou menos. Para o tempo de aplicação $1 / 3$ da amostra recebeu o selante há 6 meses. Pouco me- nos de $2 / 3$ da amostra receberam Fluroshield como material restaurador, sendo o isolamento relativo o mais prevalente $(72,2 \%$ IC $95 \% 49,1 ; 87,5)$. Mais de $2 / 3$ da amostra não apresentaram cárie (88,9\% IC95\% 67,2;96,9) (Tabela 1). Para as associações entre alguma perda de retenção e variáveis sociodemográficas, não foram encontradas diferenças significativas.

Tabela 1 - Descrição das variáveis sociodemográficas, prevalência de cárie e alguma perda de retenção dos selantes de pacientes da clínica de Odontologia da Unisul que receberam selantes há 6 meses $(n=18)$

\begin{tabular}{|c|c|c|c|}
\hline Variável & $N$ & $\%$ & IC 95\% \\
\hline \multicolumn{4}{|l|}{$\operatorname{Sexo}(n=18)$} \\
\hline Masculino & 5 & 27,8 & 12,$5 ; 50,9$ \\
\hline Feminino & 13 & 72,2 & 49,$1 ; 87,5$ \\
\hline \multicolumn{4}{|l|}{ Idade em anos $(n=18)$} \\
\hline $8-12$ & 6 & 33,3 & 16,$3 ; 56,3$ \\
\hline $13-19$ & 4 & 22,2 & 9,$0 ; 45,2$ \\
\hline$\geq 20$ & 8 & 44,4 & 24,$6 ; 66,3$ \\
\hline \multicolumn{4}{|c|}{ Renda em reais $(R \$)(S M)(n=17)$} \\
\hline$\geq 2.810(\geq 3 \mathrm{SM})$ & 6 & 33,3 & 16,$3 ; 56,3$ \\
\hline $2.810-1.874(2-3 \mathrm{SM})$ & 5 & 27,8 & 12,$5 ; 50,9$ \\
\hline$<1.874(<2 \mathrm{SM})$ & 6 & 33,3 & 16,$3 ; 56,3$ \\
\hline \multicolumn{4}{|l|}{ Tempo de aplicação $(n=18)$} \\
\hline 6 meses & 6 & 33,3 & 16,$3 ; 56,3$ \\
\hline 7-8 meses & 1 & 5,6 & 1,$0 ; 25,8$ \\
\hline$>9$ meses & 11 & 61,1 & 38,$6 ; 79,7$ \\
\hline \multicolumn{4}{|c|}{ Material de aplicação $(n=18)$} \\
\hline Fluroshield & 11 & 61,1 & 38,$6 ; 79,7$ \\
\hline Flow & 7 & 38,9 & 20,$3 ; 61,4$ \\
\hline \multicolumn{4}{|l|}{ Tipo de isolamento } \\
\hline Absoluto & 5 & 27,8 & 12,$5 ; 50,9$ \\
\hline Relativo & 13 & 72,2 & 49,$1 ; 87,5$ \\
\hline \multicolumn{4}{|c|}{ Alguma perda de retenção $(n=18)$} \\
\hline Não & 9 & 50,0 & 29,$0 ; 71,0$ \\
\hline Sim & 9 & 50,0 & 29,$0 ; 71,0$ \\
\hline \multicolumn{4}{|c|}{ Prevalência de cárie dentária $(n=18)$} \\
\hline Não & 16 & 88,9 & 67,$2 ; 96,9$ \\
\hline Sim & 2 & 11,1 & 3,$1 ; 32,8$ \\
\hline
\end{tabular}

Fonte: autores.

Foram avaliados 57 dentes, dos quais 40 apresentavam retenção total $(70,2 \%)$. Para a perda de retenção do selante de $1 / 3$ da superfície dentária (R1), 9 dentes foram encontrados (15,8\%). Segundo avaliação para perda de retenção do selante de 2/3 da superfície dentária (R2), foram observados 4 dentes $(7,0 \%)$. Segundo associação entre retenção total e perdas de retenção e as variáveis tempo e material de aplicação, tipo de isolamento e prevalência de cárie, observou-se associação significativa entre dentes com retenção total e ausência de cárie $(77,8 \%)$ e isolamento relativo $(76,5 \%)$. Para a associação da retenção $\mathrm{R} 1$ e variáveis, $50 \%$ dos dentes que apresentaram esse tipo de perda foram isolados com isolamento absoluto quando comparados ao relativo (12\%). Para perda total, a associação significativa com cárie foi observada em $22,2 \%$ dos dentes que tiveram perda total quando comparados ao seu par $(4,2 \%)$ (Tabela 2$)$. 
Tabela 2 - Associação entre número de dentes com retenção total, parciais 1 e 2, perda total e tempo e material de aplicação, prevalência de cárie e tipo de isolamento de pacientes da clínica de Odontologia da Unisul que receberam selantes há 6 meses ( $n=57$ )

\begin{tabular}{|c|c|c|c|c|c|c|c|c|}
\hline \multirow[b]{2}{*}{ Variável } & \multicolumn{2}{|c|}{ Retenção total } & \multicolumn{2}{|c|}{ Retenção parcial R1 } & \multicolumn{2}{|c|}{ Retenção parcial R2 } & \multicolumn{2}{|c|}{ Perda total } \\
\hline & $\begin{array}{c}\% \\
\text { (IC 95\%) }\end{array}$ & $\mathrm{p}^{*}$ & $\begin{array}{c}\% \\
\text { (IC 95\%) } \\
\end{array}$ & $\mathrm{p}^{*}$ & $\begin{array}{c}\% \\
\text { (IC 95\%) }\end{array}$ & $p^{*}$ & $\begin{array}{c}\% \\
\text { (IC 95\%) }\end{array}$ & $\mathrm{p}^{*}$ \\
\hline Tempo de aplicação $(n=18)$ & & 0,5044 & & 0,9018 & & 0,4111 & & 0,1588 \\
\hline 6 meses & $\begin{array}{c}77,8 \\
(54,8 ; 91,0)\end{array}$ & & $\begin{array}{c}16,7 \\
(5,8 ; 39,2)\end{array}$ & & $\begin{array}{c}11,1 \\
(3,1 ; 32,8)\end{array}$ & & $\begin{array}{c}0,0 \\
(0,0 ; 17,6)\end{array}$ & \\
\hline$>6$ meses & $\begin{array}{c}69,2 \\
(53,6 ; 81,4) \\
\end{array}$ & & $\begin{array}{c}15,4 \\
(7,2 ; 29,7) \\
\end{array}$ & & $\begin{array}{c}5,1 \\
(1,4 ; 16,9) \\
\end{array}$ & & --- & \\
\hline Material de aplicação $(n=18)$ & & 0,8879 & & 0,6024 & & 0,9506 & & 0,2151 \\
\hline Fluroshield & $\begin{array}{c}71,4 \\
(56,4 ; 82,8)\end{array}$ & & $\begin{array}{c}14,3 \\
(6,7 ; 27,8)\end{array}$ & & $\begin{array}{c}7,1 \\
(2,5 ; 19,0)\end{array}$ & & $\begin{array}{c}9,5 \\
(3,8 ; 22,1)\end{array}$ & \\
\hline Flow & $\begin{array}{c}73,3 \\
(48,1 ; 89,1) \\
\end{array}$ & & $\begin{array}{c}20,0 \\
(7,1 ; 45,2) \\
\end{array}$ & & $\begin{array}{c}6,7 \\
(1,2 ; 29,8) \\
\end{array}$ & & $\begin{array}{c}0,0 \\
(0,0 ; 20,4) \\
\end{array}$ & \\
\hline Tipo de isolamento $(n=18)$ & & 0,0261 & & 0,0151 & & 0,3280 & & 0,4768 \\
\hline Absoluto & $\begin{array}{c}33,3 \\
(9,7 ; 70,0)\end{array}$ & & $\begin{array}{c}50,0 \\
(18,8 ; 81,2)\end{array}$ & & $\begin{array}{c}16,7 \\
(3,0 ; 56,4)\end{array}$ & & $\begin{array}{c}0,0 \\
(0,0 ; 39,0)\end{array}$ & \\
\hline Relativo & $\begin{array}{c}76,5 \\
(63,2 ; 86,0) \\
\end{array}$ & & $\begin{array}{c}11,8 \\
(5,5 ; 23,4) \\
\end{array}$ & & $\begin{array}{c}5,9 \\
(2,0 ; 15,9) \\
\end{array}$ & & $\begin{array}{c}7,8 \\
(3,1 ; 18,5) \\
\end{array}$ & \\
\hline Prevalência de cárie $(n=18)$ & & 0,0455 & & 0,5641 & & 0,6003 & & 0,0517 \\
\hline Não & $\begin{array}{c}77,8 \\
(63,5 ; 86,7)\end{array}$ & & $\begin{array}{c}14,6 \\
(7,2 ; 27,1)\end{array}$ & & $\begin{array}{c}6,3 \\
(2,2 ; 16,8)\end{array}$ & & $\begin{array}{c}4,2 \\
(1,2 ; 14,0)\end{array}$ & \\
\hline Sim & $\begin{array}{c}44,4 \\
(18,9 ; 73,3) \\
\end{array}$ & & $\begin{array}{c}22,2 \\
(6,3 ; 54,7) \\
\end{array}$ & & $\begin{array}{c}11,1 \\
(1,2 ; 43,5) \\
\end{array}$ & & $\begin{array}{c}22,2 \\
(6,3 ; 54,7) \\
\end{array}$ & \\
\hline
\end{tabular}

*Teste Qui-Quadrado.

Fonte: autores.

Os dentes mais acometidos pela perda de retenção, em ordem decrescente, foram: segundo molar superior esquerdo; primeiro molar superior esquerdo; segundo molar inferior direito; primeiro molar inferior direito. Menor proporção de alguma perda de retenção foi encontrada no primeiro molar inferior esquerdo (Figura 1).

Figura 1 - Distribuição percentual dos dentes com alguma perda de retenção de selantes aplicados em pacientes da clínica de Odontologia da Unisul, após, pelo menos, 6 meses da aplicação (n=57)

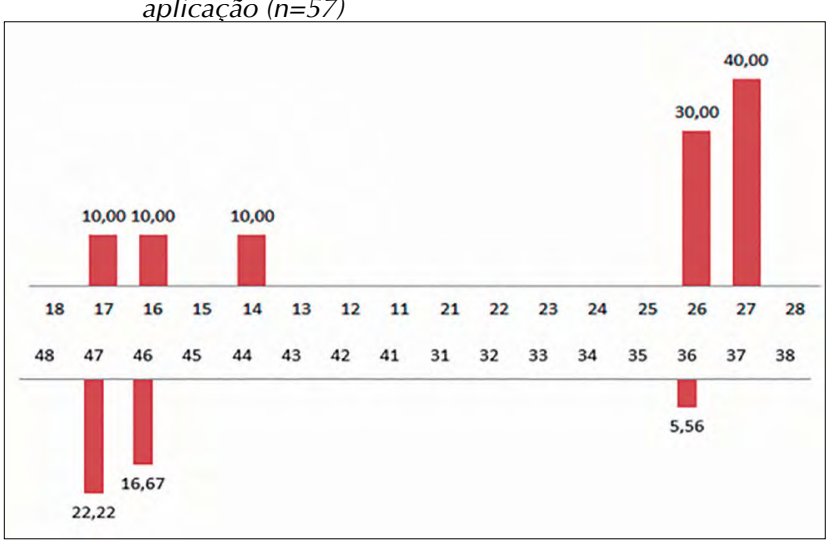

Fonte: autores.

De outro modo, maiores proporções de retenção total foram encontradas nos primeiros e nos segundos molares inferiores esquerdos. Menores proporções de retenção foram encontradas nos primeiros e nos segundos molares superiores esquerdos e nos segundos molares (Figura 2).
Figura 2 - Distribuição percentual dos dentes com retenção total dos selantes aplicados em pacientes da clínica de Odontologia da Unisul, após, pelo menos, 6 meses da aplicação $(n=57)$

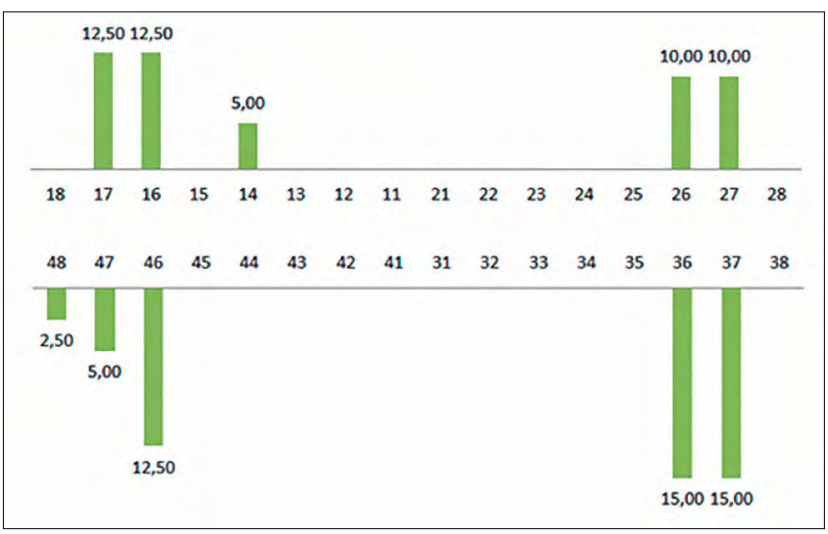

Fonte: autores.

Associação significativa entre alguma perda de retenção entre os dentes e isolamento do campo operatório foi observada neste estudo. Possuir isolamento relativo não foi indicador de perda de alguma retenção quando comparado ao isolamento absoluto (Figura 3). 
Figura 3 - Distribuição percentual de alguma perda de retenção entre os dentes e os tipos de isolamento do campo operatório $(n=57)$

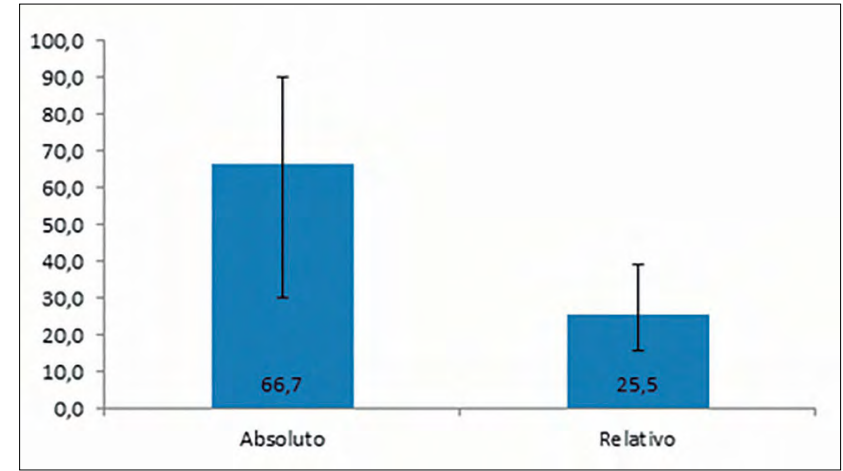

Nota: teste Qui-Quadrado ( $p=0,0370)$.

Fonte: autores.

\section{Discussão}

Dos prontuários avaliados, um número absoluto reduzido de pacientes recebeu selamento, dentre eles, a faixa de crianças e adolescentes, do sexo feminino e com renda inferior a três salários mínimos. Da mesma maneira, observou-se uma maior utilização dos serviços da universidade por indivíduos de menor renda. $\mathrm{O}$ isolamento relativo e o selante resinoso Fluroshield foram a técnica e o material mais utilizados. Metade da amostra perdeu alguma retenção, embora, em porcentagem de número de dentes avaliados, $70 \%$ da amostra tenham apresentado dentes com retenção total; assim como uma baixa experiência de cárie foi observada.

A utilização de serviços por mulheres é discutida na literatura, uma vez que homens e mulheres possuem diferentes atitudes quanto ao comportamento em saúde, e mulheres utilizam mais o serviço, percebem mais a dificuldade de mastigação, a dor dentária e a necessidade de tratamento ${ }^{18,19}$.

Segundo a literatura, as condições socioeconômicas sinalizam as necessidades de tratamento e utilização dos serviços, isso significa que baixos estratos de renda e escolaridade da mãe são indicativos de maiores necessidades, em especial quando considerada a cárie dentária ${ }^{20}$. Para o presente estudo, as associações não foram significativas, embora mais da metade da amostra tenha sido composta de pacientes com renda inferior a três salários mínimos.

Sob a perspectiva das faixas etárias, é esperado que um maior número de selantes seja aplicado em crianças e adolescentes, pelo seu potencial preventivo. Segundo estudo ecológico realizado com adolescentes no estado de São Paulo, entre os anos de 1998 e $2002^{21}$, houve diminuição das necessidades de restaurações (de uma e de duas ou mais superfícies) e aumento das necessidades de selantes, tal achado reflete uma redução da gravidade da cárie dentária, embora ainda prevalente pela necessidade do selante. Sabe-se, também, que o período da adolescência é permeado pela falta de cuidados com a saúde, assim, os adolescentes tendem a negligen- ciar os cuidados das práticas preventivas em saúde bucal, o que reflete na necessidade de intervenção ${ }^{21}$.

Em relação ao tipo de isolamento, observou-se uma maior proporção de dentes com retenção total que receberam o isolamento relativo. Embora reconheça-se o isolamento absoluto como a melhor opção, o isolamento relativo tem mostrado resultados significativos no padrão de retenção ${ }^{22}$ e longevidade dos materiais odontológicos ${ }^{23}$.

Embora o selante Fluroshield tenha tido uma proporção de perda de 55\%, segundo estudo realizado na Universidade Federal de Campina Grande, com 40 indivíduos que receberam Fluroshield, 92,5\% tiveram retenção total ${ }^{24}$. Em outro estudo, o material Fluroshield apresentou efetividade de $100 \%$, e não foi observada incidência de lesão de cárie nos molares selados após 12 meses de acompanhamento $^{25}$.

Para este estudo, $70 \%$ dos dentes apresentavam padrões adequados de retenção do selante. Segundo estudo realizado por estudantes de Odontologia da Universidade Regional de Blumenau (Furb) ${ }^{13}$, os padrões de retenção de selante, quando aplicados por estudantes, estiveram em níveis abaixo deste estudo, com $31 \%$ de retenção total do selante resinoso, $6 \%$ do cimento de ionômero de vidro e $40 \%$ da resina Flow, embora a literatura ressalte não haver diferenças entre a retenção dos selantes entre profissionais especializados ou generalistas ${ }^{26}$.

Para os dentes acometidos pela cárie e que necessitaram de selamento, observou-se maior prevalência para os molares, o que corrobora a literatura sobre a suscetibilidade de cárie entre os molares ${ }^{4}$. Contudo, observou-se também uma maior perda de retenção entre os molares permanentes superiores, o que compactua com a literatura sobre a perda de retenção dos selantes nos molares ${ }^{27}$,em especial nos dentes superiores ${ }^{28}$.

Os selantes de fossas e fissuras aplicados, mesmo com perdas totais ou parciais, foram eficazes na manutenção da maioria das superfícies dentárias, que se apresentaram livres de lesão de cárie. Salienta-se a importância da periodicidade na avaliação $0^{24} \mathrm{e}$ a abordagem preventiva para os riscos da atividade cariosa, uma vez que as superfícies suscetíveis tendem a permitir o acúmulo de biofilme dentário e maiores riscos ${ }^{29}$.

Segundo a literatura, faz-se necessário acompanhar com maior rigor as aplicações de selantes de fossas e fissuras feitas por estudantes de graduação em Odontologia ${ }^{13}$. Considerando o fator aprendizagem, os autores destacam a importância na atenção para as condutas de planejamento, no sentido da indicação clínica, do acompanhamento e da técnica de aplicação, em especial para os dentes com maiores desafios de retenção.

É importante destacar que as associações não significativas se atribuem à amostra reduzida de pacientes da clínica de Odontologia da Unisul que receberam selantes, o que pode ser considerada 
uma limitação do estudo. Todavia, pode-se atribuir o número reduzido de pacientes que receberam selante, pela correta indicação do selamento dos dentes, em casos de vulnerabilidade para o agravo bucal cárie dentária. A baixa prevalência de cárie pode ser justificada pelo período de avaliação de 6 a 9 meses para o estudo, sugerindo a necessidade de monitoramento desses pacientes.

\section{Conclusão}

Para o presente estudo, verificou-se que a utilização do roteiro validado pela literatura auxiliou na avaliação e na análise do padrão de retenção dos selantes de fossas e fissuras, sendo este recomendado. Uma parcela importante dos selamentos foi realizada com isolamento relativo, e, em termos proporcionais, observou-se que quase $2 / 3$ dos dentes se mantiveram selados. Embora os dentes selados com resina Flow e isolamento absoluto tenham apresentado retenção total no período avaliado, sugere-se a realização de mais estudos de efetividade da aplicação e avaliação de selantes com isolamento relativo.

Não obstante, a proporção de indivíduos com alguma perda de retenção foi importante, e isso pode se refletir no processo de aprendizagem do estudante, assim como na sua habilidade de aplicação em determinados dentes. Além disso, verificou-se que, pela associação estatística, mais de $2 / 3$ dos dentes avaliados com retenção total não tinham cárie, o que permite concluir que os selantes de fossas e fissuras, aplicados por estudantes, foram eficazes na preservação da maioria das superfícies dentárias livres de cárie.

\section{Abstract}

Objective: to evaluate the effectiveness of pit and fissure sealants applied by undergraduate dental students of the University of Southern Santa Catarina (Unisul), SC, Brazil, after 6 months, and to estimate the prevalence of caries in the participants. Subjects and method: observational and descriptive study with individuals from the dental clinics. The effectiveness of pit and fissure sealants was evaluated using a script validated in the literature, and the prevalence of caries was dichotomized. The data were analyzed by descriptive statistics and the associations were made using the chi-square test. Results: the response rate of the study was $94.7 \%(n=18)$, $2 / 3$ of the sample consisted of women $(72.2 \% 95 \% \mathrm{Cl}$ $49.1,87.5), 60 \%$ of the patients received three Brazilian minimum wages or less, and a great portion of the participants had sealed their teeth at least 9 months earlier. A little under 2/3 of the sample received Fluroshield as restorative material for their teeth. Among the 57 teeth evaluated, $70.2 \%$ presented complete retention. The teeth most affected by loss of retention were maxillary left second molars (40\%). More than $2 / 3$ of the sample did not present dental caries $(88.9 \% 95 \% \mathrm{Cl}, 67.2$, 96.9). Relative isolation was the most prevalent $(72.2 \%$ $95 \% \mathrm{Cl} 49.1$, 87.5). Conclusion: from the perspective of the practices of dental students, half of the sample of patients presented some loss of sealant retention, despite the greater number of complete retentions and absence of recurrent caries. This reflects the importance of the teacher-student relationship in the planning and correct application of sealing techniques by dental students.

Keywords: Effectiveness. Oral health. Pit and fissure sealants. Dental caries susceptibility.

\section{Referências}

1. Brasil. Ministério da Saúde. Coordenação Nacional de Saúde Bucal. Secretaria de Atenção à saúde. Programa Nacional de Saúde Bucal. SB BRASIL 2010: Resultados principais. Brasília: Ministério da Saúde; 2011.

2. Guedes-Pinto AC, Bönecker M, Rodrigues CRMD (Org.). Fundamentos de Odontologia. São Paulo: Santos; 2009.

3. Ahovuo-Saloranta A, Forss H, Hiiri A, Nordblad A, Mäkelä M. Pit and fissure sealants versus fluoride varnishes for preventing dental decay in the permanent teeth of children and adolescents. Cochrane Database Syst Rev 2016; 74(2):171-6.

4. Batchelor PA, Sheiham A. Grouping of tooth surfaces by susceptibility to caries: a study in 5-16 year-old children. BMC Oral Health 2004; 4(2):1-6.

5. Powell LV. Caries prediction: a review of the literature. Community Dent Oral Epidemiol 1998; 26(6):361-71.

6. Skeie MS, Raadal M, Strand GV, Espelid I. The relationship between caries in the primary dentition at 5 years of age and permanent dentition at 10 years of age - a longitudinal study. Int J Paediatr Dent 2006; 16(3):152-60.

7. Simonsen RJ. Retention and effectiveness of dental sealant after 15 years. JADA 1991; 122:34-42.

8. Pinto VG. Saúde bucal coletiva. 6. ed. São Paulo: Santos; 2013.

9. Mejare I, Lingztro MP, Peterzzon LG, Holm AK, Twetman $\mathrm{S}$, Kalleztal G, et al. Caries-preventive effect of fissure sealants: a systematic review. Acta Odontol Scand 2003; 6(6):321-03.

10. Aguilar FG, Drubi-Filho B, Casemiro LA, Watanabe MG, Pires-de-Souza FC. Retention and penetration of a conventional resin-based sealant and a photochromatic flowable composite resin placed on occlusal pits and fissures. J Indian Soc Pedod Prev Dent 2007; 25:169-73.

11. Busato AS, Maltz M. (Org.). Cariologia: aspectos de Dentística restauradora. São Paulo: Artes Médicas; 2014.

12. Cabral RN. Avaliação do risco à cárie em escolares na faixa etária de 5-7 anos e da efetividade do uso de selantes na prevenção de lesões cariosas em molares permanentes recém-irrompidos, 2013. 85 f. [Dissertação de Mestrado em Ciências da Saúde]. Brasília: Universidade de Brasília; 2013.

13. Rastelli MS, Reinke SMG, Scalabrin M, Santos FA. Avaliação dos selantes de fossas e fissuras aplicados por estudantes de Odontologia. Rev Odontologia Unesp 2012; 41(5):324-9.

14. Crivello Junior O, Antunes JLF, Peres MA. (Org.). Epidemiologia da saúde bucal. Rio de Janeiro: Guanabara Koogan; 2010.

15. Basting RT, Cerqueira AMC, Pereira AC, Meneghim MC, Corrente JE. Avaliação clínica de uma resina composta modificada por poliácido, utilizada como selante oclusal, quando aplicada por dentista, THD e graduando. Rev Odontol Univ São Paulo 1999; 13(2):111-7. 
16. Ismail AI, Sohn W, Tellez M, Amaya A, Sen A, Hasson H, et al. The International Caries Detection and Assessment System (ICDAS): an integrated system for measuring dental caries. Community Dent Oral Epidemiol 2007; 35:170-8.

17. International Caries Detection and Assessment System (ICDAS) Coordinating Committee. Criteria Manual - International Caries Detection and Assessment System (ICDAS II). Scotland: Dental Health Services Research Unit; 2005 [citado 2016 ago. 2017]. Disponível em URL: http://www.icdas.org.

18. Figueiredo DR, Peres MA, Luchi CA, Peres KG. Fatores associados à dificuldade para mastigar em adultos no sul do Brasil. Rev Saúde Pública 2013; 47(6):1028-38.

19. Farghaly JG, Fachin LV, Otton R, Guaré RO, Leite MF. Efeito do gênero (masculino e feminino) sobre a cárie dentária e parâmetros salivares de crianças. Pesq Bras Odontoped Clin Integr 2013; 13(1):11-5.

20. Peres MA, Bastos AJ, Peres KG, Araújo CLP, Menezes AMB. Life course dental caries determinants and predictors in children aged 12 years: a population-based birth cohort. Community Dent Oral Epidemiol 2009; 37(2):123-33.

21. Gushi LL, Rihs LB, Soares MC, Forni TIB, Vieira V, Wada $\mathrm{RS}$, et al. Cárie dentária e necessidades de tratamento em adolescentes do estado de São Paulo, 1998 e 2002. Rev Saúde Pública 2008; 42(3):480-6.

22. Garbin CAS, Santos KT, Garbin AJI, Pizzatto E, Moroso TT. Retenção de selante de fóssulas e fissuras sob três tipos de isolamento. Pesq Bras Odontoped Clín Integr 2008; 8(2):175-8.

23. Pallesen U, van Dijken JW, Halken J, Hallonsten AL, Höigaard R. Longevity of posterior resin composite restorations in permanent teeth in Public Dental Health Service: a prospective 8 years follow up. J Dent 2013; 41:297-306.

24. Albuquerque ACL, Araújo OMC, Silva DF, Pereira ARB. Avaliação clínica do custo/benefício de selantes oclusais. Rev Saúde e Ciência 2014; 3(1):69-80.

25. Sgavioli CAPP. Avaliação clínica do tempo de permanência de um selante de fossas, cicatrículas e fissuras oclusais, sem ou com posterior aplicação tópica de flúor. $175 \mathrm{f}$. [Dissertação de Mestrado em Odontologia]. Bauru: Universidade de São Paulo; 2000. Disponível em URL: http://www.teses.usp. br/teses/disponiveis/25/25131/tde-11082005-093105/publico/ ClaudiaSgavioli.pdf.

26. Ferguson FS, Ripa LW. Evaluation of the retention of two sealants applied by dental students. J Dent Educ 1980; 44:494-6.

27. Andrade APRCB, Imparato JCP, Momesso MGC, Silva SREP, Romancini DDA, Doenha MMAS. Retenção do selante ionomérico em molares na fase de irrupção na primeira infância. Rev Assoc Paul Cir Dent 2015; 69(4):340-4.

28. Guimarães AO. Avaliação da eficácia de selante ionomérico modificado por resina e escovação dentária supervisionada na prevenção de cárie oclusal. 2005. 61 f. [Dissertação de Mestrado em Odontologia Preventiva e Social]. Natal: Universidade Federal do Rio Grande do Norte; 2005. Disponível em URL: https://repositorio.ufrn.br/jspui/bitstream/123456789/17035/1/AmandaOG.pdf.

29. Jiang S, Chen S, Zhang C, Zhao X, Huang X, Cai Z. Effect of the Biofilm Age and Starvation on Acid Tolerance of Biofilm Formed by Streptococcus mutans Isolated from CariesActive and Caries-Free Adults. Int J Mol Sci 2017; 18(4):713.

\section{Endereço para correspondência:}

Viviane Silveira Marques

Avenida Pedra Branca, 25, Cidade Universitária Pedra Branca

88137-900, Palhoça, SC, Brasil

Telefone: (48) 999025146

E-mail: marques.viviane@unisul.br

Recebido: 22/06/18. Aceito: 30/07/18. 